\title{
ESTRUCTURA DE UN BOSQUE PLANTADO CON PROPÓSITO DE REHABILITACIÓN EN CHÍA (CUNDINAMARCA, COLOMBIA)
}

Fecha de recepción: 19 de abril de 2016 • Fecha de Evaluación: 18 de junio de 2016 • Fecha de aceptación: 2 de julio de 2016 - Disponible en línea: 25 de julio de 2016

\section{STRUCTURE OF A PLANTED FOREST WITH PURPOSE OF REHABILITATION IN CHIA (CUNDINAMARCA, COLOMBIA)}

Darío Pérez', Juan Carlos Barbosa², Sandra Pilar Cortés S. ${ }^{3}$

\section{RESUMEN}

Este estudio se efectuó en el año 2009 en un área de 2 ha sobre la cual, dieciséis años atrás, el Profesor Thomas van der Hammen realizó la siembra de especies nativas, queriendo recrear un bosque de planicie típico de los alrededores de la Sabana de Bogotá. La caracterización estructural de la vegetación se realizó teniendo en cuenta el establecimiento de intervalos de clase en los parámetros de abundancia, altura, DAP y cobertura. Para caracterizar los tipos de vegetación con respecto a atributos seleccionados, se calculó el IPF, el IVI y el VIF. Como resultado se encontró la consolidación de los estratos arbóreo y arbustivo y el establecimiento de una estructura vegetal comparables a la de los bosques andinos circundantes. El proyecto diseñado por el Profesor Thomas van der Hammen convirtió un área degradada en un parche resiliente de vegetación que, hoy día y luego de relativamente poco tiempo, permite probar que las áreas restauradas pueden servir como conectores de los remanentes de vegetación original, siendo un ejemplo vivo de la posibilidad de recuperar un ecosistema a partir de la relación recíproca entre sociedades y naturaleza.

Palabras clave: Bosque andino, Resiliencia, Sabana de Bogotá, Thomas van der Hammen, Restauración ecológica.

Biólogo, Magíster en Geografía. Universidad Nacional de Colombia,

2 Biólogo, Especialista en Evaluación del Impacto Ambiental de Proyectos. Universidad de Bogotá Jorge Tadeo Lozano, juank.barbosa@gmail.com

3 Bióloga, Doctorado en Ciencias Biológicas. Universidad Nacional de Colombia, sanpicor@yahoo.com

4 Autor corresposal. E-mail: daaperezgo@unal.edu.co 


\section{ABSTRACT}

This research was realized in the year 2009, in an area of two hectares on which, sixteen years ago, Professor Thomas van der Hammen made the planting of native species in nursery stage, wanting to recreate a forest of plain typical around the Sabana de Bogotá. The structural characterization of the vegetation was made taking into account the establishment of class intervals on the parameters of abundance, height, DAP and coverage. To characterize the types of vegetation with respect to selected attributes, the IPF, the IVI and the VIF was calculated. As a result, the consolidation of tree and shrub and the establishment of a plant structure comparable to that of the surrounding Andean forests were found. The project designed by Professor Thomas van der Hammen became a degraded area in a resilient patch of vegetation that, today and after a relatively short time, can prove that the restored areas can serve as connectors remnants of original vegetation, been an example of the possibility to recover an ecosystem from the interaction between societies and nature.

Key words: Andean forest, Resilience, Sabana de Bogotá, Thomas van der Hammen, Ecological restoration.

\section{INTRODUCCIÓN}

Colombia se halla ubicada en la esquina noroccidental de Suramérica, es un país con grandes contrastes geográficos que posee ecosistemas desde los bosques húmedos y calientes hasta los desiertos, las costas y los páramos (Pérez-Arbeláez 1959). El municipio de Chía presenta una precipitación anual aproximada de $750 \mathrm{~mm}$. El régimen de distribución de lluvias es bimodal, con épocas secas entre Junio - Septiembre y Diciembre - Marzo. Según Claro (1995), teniendo en cuenta el balance hídrico, se observa un déficit de agua en el suelo durante las épocas secas y en las húmedas un almacenamiento, sin escorrentía. El clima se puede clasificar como semiseco, con una temperatura media anual de entre 11 y $13^{\circ} \mathrm{C}$ (Cortés et al. 1999) con presencia de suelos de origen humífero del tipo Entisol-Inceptisol, en el sur, y de origen volcánico del tipo Entisol-Andisol, en el norte (Cortés et al. 1999).
El municipio de Chía, al igual que los otros municipios de la Sabana, fue declarado legalmente como de "interés ecológico nacional" según lo dispuesto en el artículo 61 de la Ley 99 de 1993 (MAVDT, 1999); es considerado por su cercanía a la ciudad de Bogotá D. C., como centro alternativo de desarrollo urbano del Distrito Capital, para lo cual existen planteamientos que enriquecen la ampliación de conceptos como: oferta ambiental, municipio verde, productor alternativo de alimentos y promotor de actividades económicas complementarias a Bogotá; que, a corto plazo, promueven vías para preservar y proteger al municipio como un ecosistema estratégico (POT, 2000).

La Sabana de Bogotá durante los últimos 60 años ha sufrido una transformación del 70\% de los ecosistemas naturales con los que contaba, reflejándose en una continua degradación en donde se 
intercambian con gran rapidez los lugares ocupados por la vegetación nativa por centros urbanísticos y sistemas agropecuarios tecnificados de acelerado crecimiento, en respuesta a las dinámicas de mercado, que no permiten la recuperación de los hábitats naturales conduciendolos a su destrucción (Van der Hammen, 1999).

La degradación ecosistémica ocurre a una magnitud y velocidad que impide el mantenimiento de su resiliencia (Saunders et al, 1991; Samper, 1999; Brasher, 2003) y reduce la biodiversidad de los bosques andinos, originando mosaicos de hábitat en donde los procesos ecológicos para el mantenimiento de ésta, son influenciados por los procesos culturales (Lozano et al, 2006).

Así, la gestión de programas donde se favorezca el desarrollo local de alternativas sociales que promuevan el establecimiento de coberturas vegetales nativas no sólo asegura el retorno natural de las fuentes de agua y el restablecimiento de la biodiversidad (Rangel y Velásquez, 1997; van der Hammen, 1998) sino que favorece la configuración de un paisaje cultural en el que coevolucionan la sociedad y la naturaleza (Castellanos, 2011).

La rehabilitación de áreas degradadas por la agricultura tecnificada es una de las estrategias necesarias para lograr la recuperación de la cobertura vegetal ya que promueve la regeneración natural minimizando el manejo forestal y generando procesos que semejen y permitan recuperar parte de la estructura y la dinámica del ecosistema original al que en el tiempo se quiere llegar (Londoño y Torres, 2015).

Producto de su conocimiento acerca de la historia de la dinámica en las comunidades vegetales, en Colombia, el profesor Thomas van der Hammen instauró en un sector de la Finca "Santa Clara", un proyecto de recuperación de áreas transformadas por la actividad agropecuaria para establecer gradualmente las combinaciones florísticas que funcionen como un patrón para el direccionamiento de los procesos espontáneos de sucesión vegetal acordes a la región de la Sabana de Bogotá; de esta manera, las áreas rehabilitadas pueden ser restauradas, servir como conectores de los remanentes de vegetación original entre zonas altas y bajas $y$, a mediano plazo, lograr que "la biodiversidad atraiga biodiversidad", como lo decía el mismo profesor Thomas van der Hammen.

\section{MATERIALES Y MÉTODOS}

\section{Área de estudio}

El estudio se realizó en zona rural del municipio de Chía (Departamento de Cundinamarca, Colombia), que se encuentra en la llamada Sabana de Bogotá (van der Hammen y van Geel, 1982) a $4^{\circ} 50^{\prime} \mathrm{N}$ y $74^{\circ} 5^{\prime} \mathrm{W}$ y a una altura de 2652 m.s.n.m. La superficie del municipio es de $7.923 \mathrm{~m} 2$. Limita por el norte con el municipio de Cajicá, por el sur con el municipio de Cota y con Bogotá D.C. (localidades de Usaquén y Suba), por el oriente con Sopó y La Calera, y por el occidente con Tabio y Tenjo (POT, 2000).

El municipio de Chía presenta una precipitación anual aproximada de $750 \mathrm{~mm}$. El régimen de distribución de lluvias es bimodal, con épocas secas entre Junio - Septiembre y Diciembre - Marzo. Según Claro (1995), teniendo en cuenta el balance hídrico, se observa un déficit de agua en el suelo durante las épocas secas y en las húmedas un almacenamiento, sin escorrentía. El clima se puede clasificar como semi-seco, con una temperatura media anual de entre 11 y $13^{\circ} \mathrm{C}$. Chía, presenta suelos de origen humífero del tipo Entisol-Inceptisol, en el sur, y de origen volcánico del tipo Entisol-Andisol, en el norte (Cortés et al, 1999).

\section{Fase de campo}

Los sitios de muestreo se seleccionaron con observaciones directas en campo. Para el muestreo de la vegetación se trazaron parcelas que se 


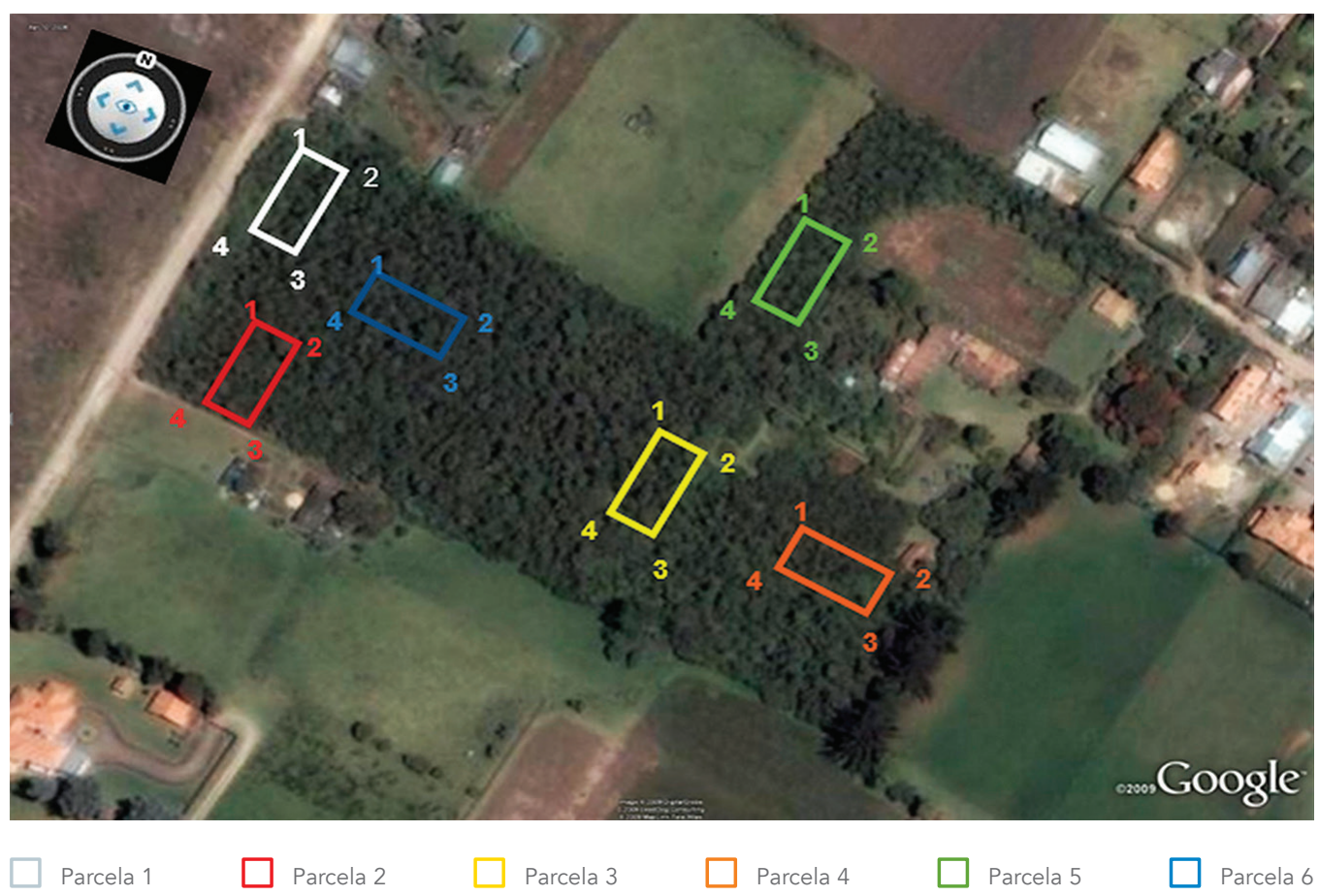

Figura 1. Vista Satelital de la ubicación de las parcelas al interior del bosque en la Finca "Santa Clara". Tomado de Google Earth ${ }^{\circledR}, 2009$.

dispusieron y orientaron según los distintos tipos de sotobosque, su grado de intervención y el criterio de siembra de especies nativas que en su momento fue realizada por el Dr. van der Hammen teniendo en cuenta la historia de la composición de la vegetación existente en ecosistemas de referencia relacionados al bosque andino bajo de la Sabana de Bogotá. Se realizaron 6 parcelas cada una de $200 \mathrm{~m}^{2}$ (Figura 1).

\section{Caracterización de la estructura \\ y composición florística}

Para analizar la distribución vertical de la vegetación, se registró la altura (por cálculo visual, en metros), el diámetro del tronco a la altura del pecho (DAP, en centímetros) y la cobertura (en metros cuadrados y en porcentaje) de los individuos de todos los estratos, que incluyó adicionalmente a los juveniles, Los registros de la altura de la vegetación se realizaron según la metodología de sugerida por Rangel y Lozano (1986), en donde la distribución correspondió a: rasante (0-0,3 m); herbáceo (>0,3$1,5 \mathrm{~m})$; arbustivo $(>1,5-5 \mathrm{~m})$; subarbóreo (arbolito) $(>5-12 m)$ y arbóreo inferior $(>12-25 m)$. De la misma manera, se tomó en cuenta lo sugerido por Rangel y Velásquez (1997) para la elaboración de los diagramas estructurales.

La caracterización estructural de la vegetación se realizó para cada levantamiento mediante el establecimiento de intervalos de clase, según los valores máximos y mínimos del conjunto total de datos obtenidos en todas las parcelas en los parámetros de abundancia, altura, DAP y cobertura. Para conocer la dominancia energética de los estratos de la 
vegetación, para este caso, también se realizaron diagramas estructurales en donde se estableció el porcentaje de cobertura en función de los estratos presentes en el área total (Rangel y Velázquez, 1997).

Por otra parte, se calculó el Índice de Predominio Fisionómico (IPF) para cada parcela, teniendo en cuenta la sumatoria de los porcentajes de cobertura relativa, área basal y densidad relativas (Rangel y Garzón, 1994). Para conocer de qué manera los conjuntos de especies aportan a procesos ecológicos que se encuentran asociados a la comunidad, como la dominancia, se calculó el índice de valor de importancia de cada especie (IVI), la frecuencia (FR) y la dominancia (DoR) relativas (Finol 1976).

Para evaluar la importancia ecológica de las familias en cada estrato de la vegetación se calculó el índice de valor de importancia para familias (VIF), todas las anteriores para los individuos con DAP $\geq 2.5$ cm, según la propuesta de Mori y Boom (1983).

Finalmente, las determinaciones taxonómicas se realizaron directamente en campo con claves taxonómicas, guías y catálogos ilustrados, para llegar hasta el nivel de especie, siguiendo la propuesta de clasificación de Judd et al. (2002).

\section{RESULTADOS}

\section{Altura}

Respecto al parámetro de altura, el bosque de planicie regenerado de la Finca 'Santa Clara' muestra como valor máximo $14 \mathrm{~m}$, el mayor porcentaje de individuos se presentó entre $0.3 \mathrm{~m}$ y $2.91 \mathrm{~m}$ de altu$\mathrm{ra}$, en donde el estrato dominante en cobertura en todos los casos fue el subarbóreo (en porcentajes mayores al 25\%), seguido del estrato arbóreo. Así mismo, los individuos que predominaron se encontraron con mayor frecuencia entre los estratos inferiores formando 11 intervalos de clase (Figuras 2 y 3 ).

\section{Cobertura}

La cobertura del bosque regenerado se distribuye en 13 intervalos de clase, en donde las coberturas menores (81\%) predominaron sobre las de mayores

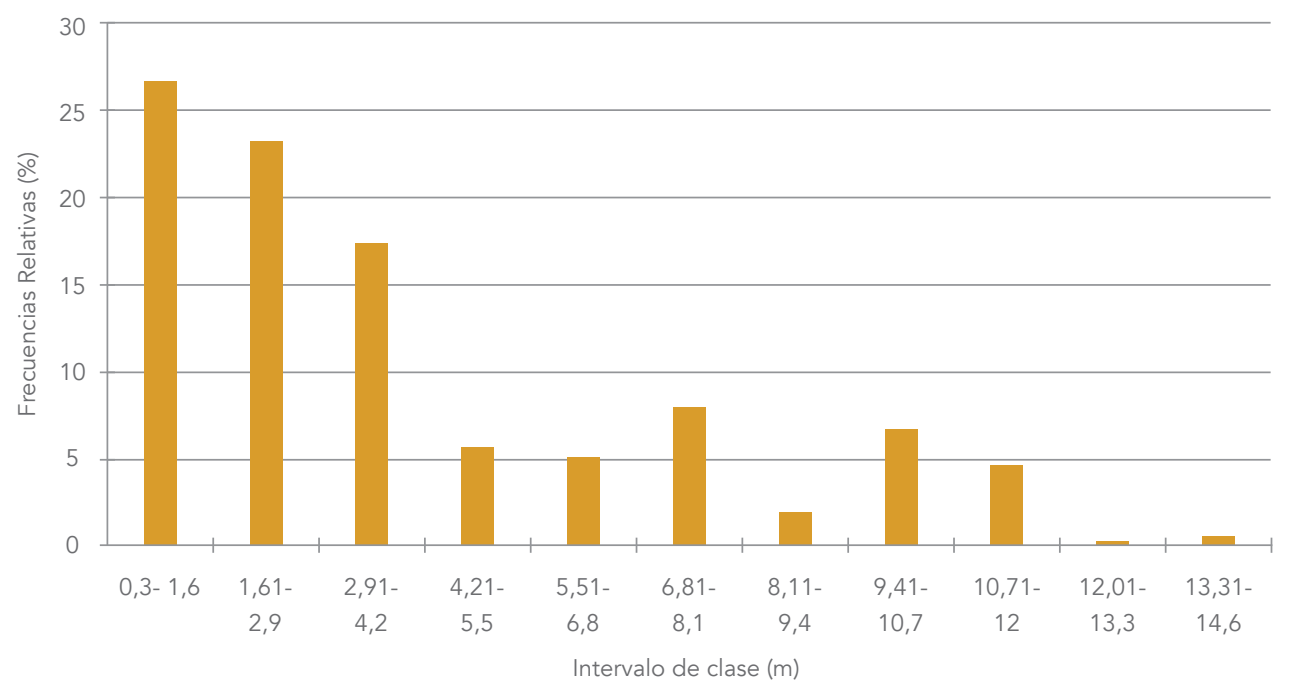

Figura 2. Distribución de clases de altura del bosque regenerado. 


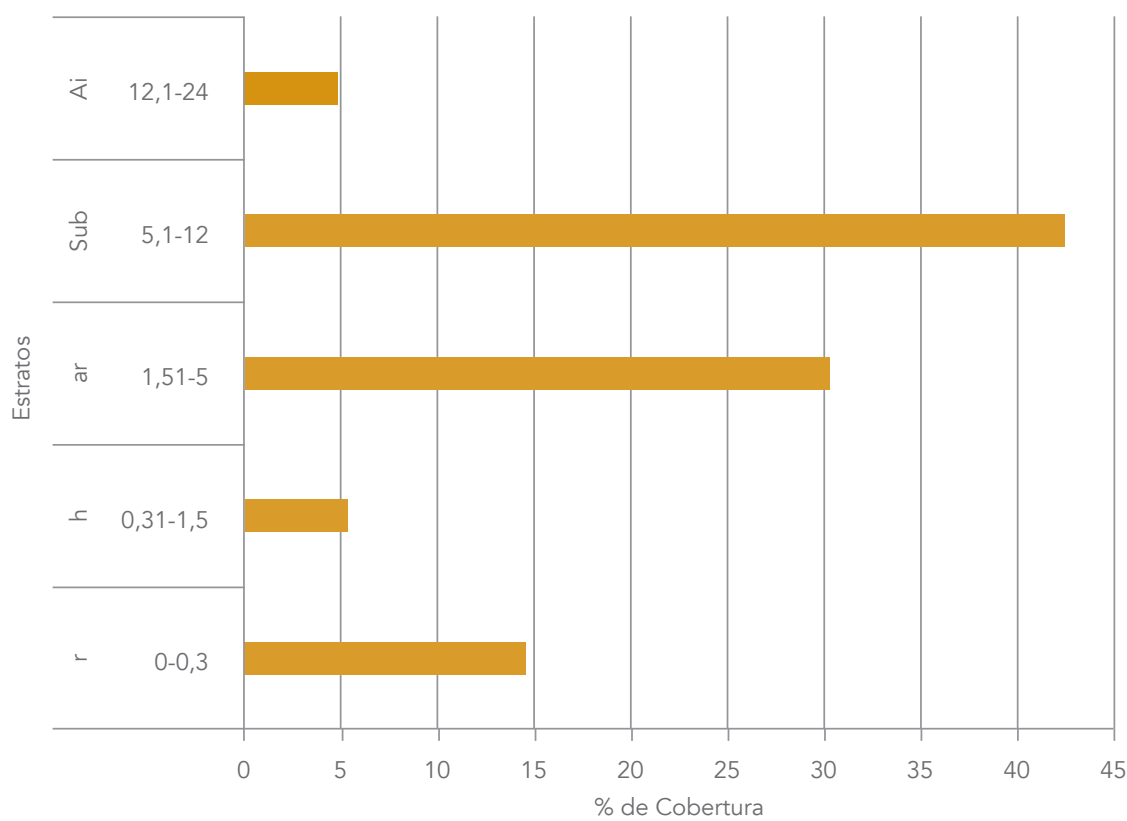

Figura 3. Distribución de cobertura por estrato del bosque regenerado.

valores, dominando el intervalo de 0.01 a $1.11 \mathrm{~m}$. La tendencia general para el bosque regenerado de la Finca "Santa Clara" fue el presentar frecuencias relativas altas en cobertura, es decir entre el 80 y el $95 \%$, en el intervalo de clase menor (0.01-1.11 m²) (Figura 4).

\section{DAP (Diámetro a la altura del pecho)}

La tendencia general, en cuanto al DAP, muestra que el intervalo de clase diamétrica de $2.5 \mathrm{~cm}$ a $8.3 \mathrm{~cm}$ representó entre el $35 \%$ y el $40 \%$ en los valores de frecuencia relativa y que, en general, las frecuencias relativas hasta la clase diamétrica con valor máximo de $22.5 \mathrm{~cm}$ están representados con valores superiores o cercanos al 10\% (Figura 5).

\section{ÍNDICES ECOLÓGICOS}

\section{Índice de Predominio Fisionómico (IPF)}

El índice de predominio fisionómico (IPF) se calculó para los individuos con DAP $\geq 2.5 \mathrm{~cm}$.
En la parcela 1 (P1), el IPF fue calculado para 21 especies; la especie con mayor valor de predominio fue Quercus humboldtii (108.05), seguido de Alnus acuminata (58.97) y Solanum ovalifolium (45.22). En el parámetro de densidad relativa la especie con mayor valor fue S. ovalifolium (31.35), seguida de $\mathrm{Q}$. humboldtii (23.24) y A. acuminata (9.19). Respecto a la cobertura el mayor valor fue $\mathrm{Q}$. humboldtii (49.83), seguido de $S$. ovalifolium (13.62) y A. acuminata (11.52). En cuanto a la dominancia relativa, la especie con mayor valor fue $A$. acuminata (38.26), seguido de Q. humboldtii (34.98) y Baccharis latifolia (11.36) (Tabla 1).

En la parcela 2 (P2) el IPF se calculó para 15 especies; el IPF más alto lo alcanzó A. acuminata (88.66) seguido de B. latifolia (54.88), y Vallea stipularis (41.12). Respecto a la densidad relativa la especie con mayor valor fue S. ovalifolium (26.72), seguida de A. acuminata (15.27) y Myrsine guianensis (9.16). En cobertura, el mayor valor fue para A. acuminata (45.65), seguido de V. stipularis (12.39) y S. ovalifolium 
(11.11). Con respecto a la dominancia relativa, la especie con mayor valor fue $B$. latifolia (41.60), seguido de A. acuminata (27.74) y V. stipularis (21.86) (Tabla 2).

En la parcela 3 (P3) el cálculo del IPF fue para 17 especies; el IPF y los valores de cobertura y dominancia relativas más altos los alcanzó A. acuminata (123, 33.81 y 63.89 , respectivamente), seguido de $V$. stipularis (58.16, 20.97 y 9.47 respectivamente). En cuanto a densidad relativa el valor más alto lo obtuvo $\mathrm{V}$. stipularis (27.71), seguido de A. acuminata (25) (Tabla 3).

En la parcela 4 (P4) se realizó el cálculo de IPF para 14 especies; los valores mayores de IPF, dominancia relativa, densidad relativa y cobertura fueron para Brugmansia sanguinea (169.50, 65.19, 48.85 y 55.51 respectivamente), seguidos de Montanoa quadrangularis $(42.79,11.32,16.67$ y 14.80 respectivamente) (Tabla 4).

Para un total de 11 especies fue calculado el valor de IPF en la parcela 5 (P5), de las cuales Citharexylum subflavescens presentó el mayor de IPF (78.49), cobertura relativa (30.68) y dominancia relativa (29.62), seguido de A. acuminata (77.33, 28.40 y 24.93, respectivamente), el cual, a su vez, presentó el mayor valor de densidad relativa (25), seguido de C. subflavescens (18.18) (Tabla 5).

El valor de IPF fue calculado para 18 especies en la parcela 6 (P6), el mayor valor de IPF fue para $S$. ovalifolium (105.75), el cual, a su vez, alcanzó los mayores valores en todos los parámetros que conducen a éste índice (39.34 de cobertura y 45.53 de densidad relativa), excepto en la dominancia relativa en donde el mayor valor fue para A. acuminata (35.53), seguido de S. ovalifolium (20.88) (Tabla 6).

\section{Índice de Valor de Importancia (IVI)}

Dentro de las especies nativas que no fueron sembradas en el bosque regenerado $S$. ovalifolium es quien alcanza el mayor valor de importancia

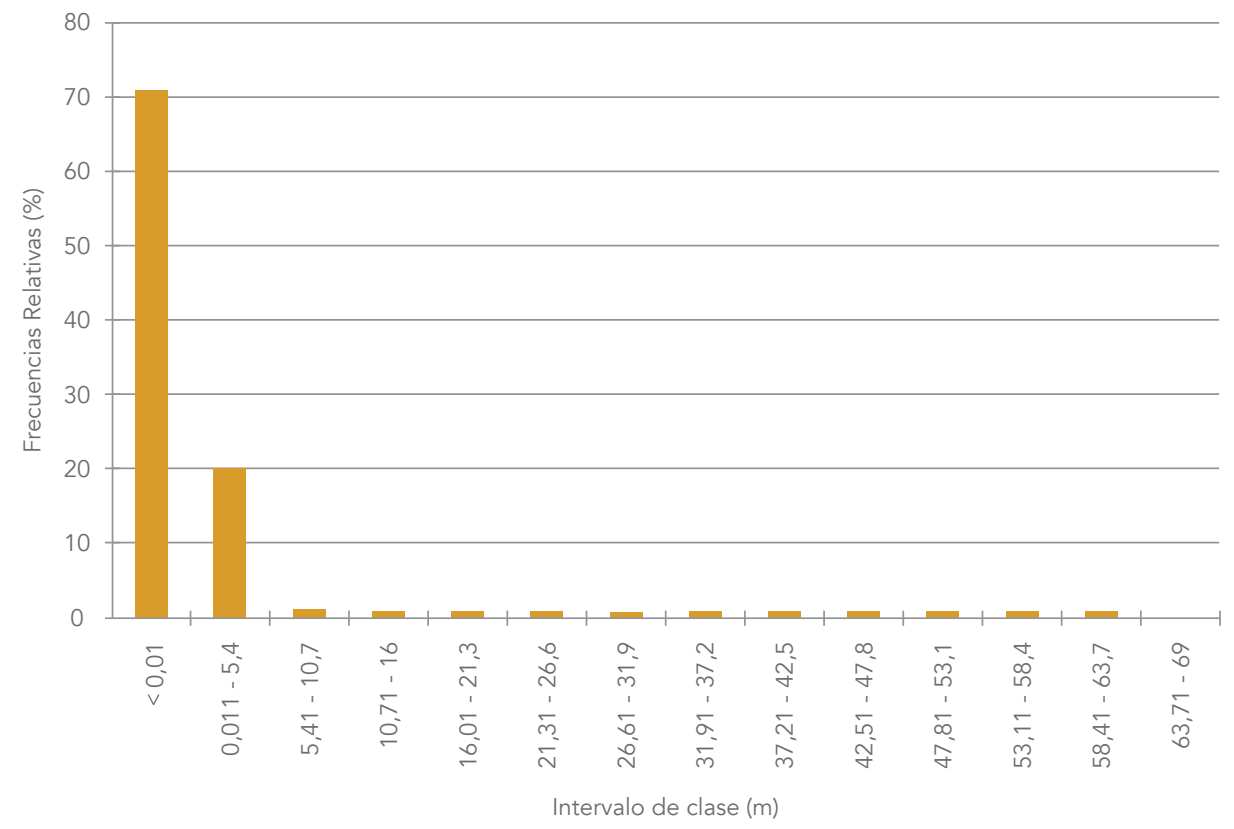

Figura 4. Distribución de clases de cobertura en el bosque regenerado. 


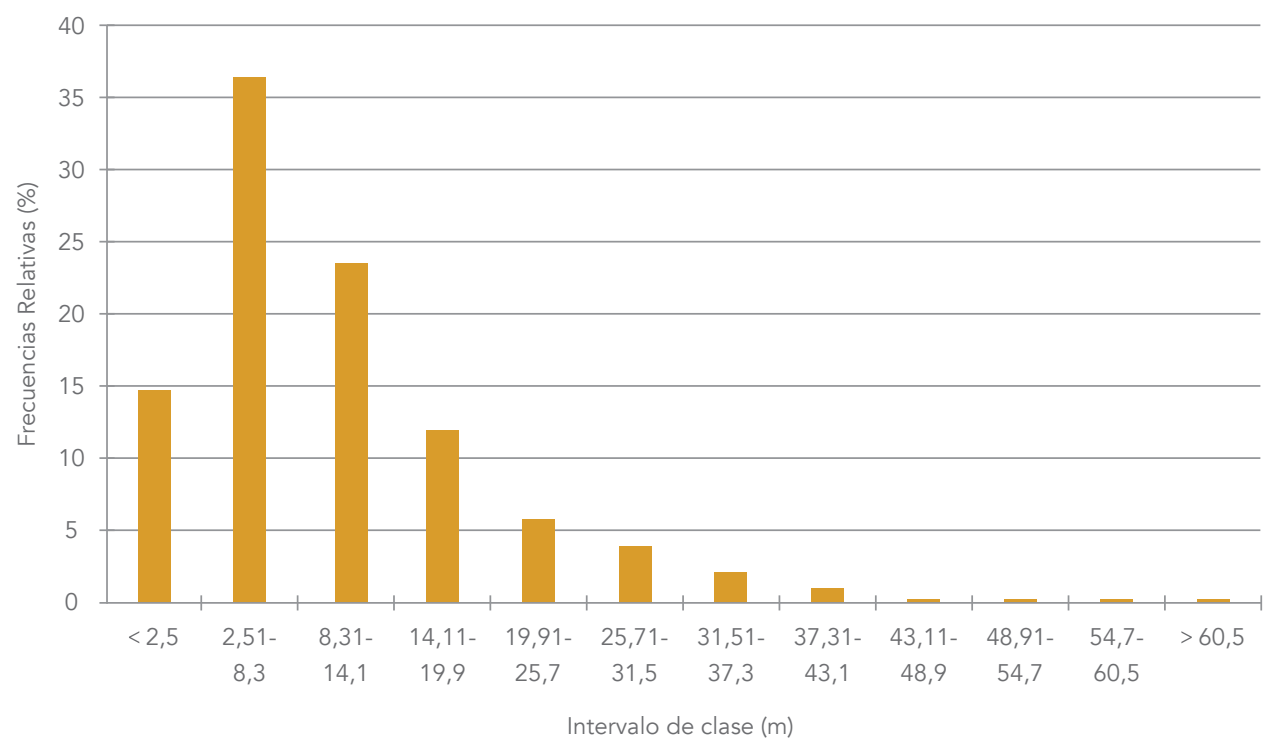

Figura 5. Distribución de clases diamétricas en el bosque regenerado.

(61.60), seguida por aquellas que fueron sembradas hace 16 años como: A. acuminata (32.51), B. sanguinea (17.79), Cestrum elegans (17.64), V. stipularis (16.65) y B. latifolia (15.49); S. ovalifolium, A. acuminata y $B$. sanguinea, alcanzan los mayores valores de densidad relativa seguida por $A$. acuminata la cual, junto con $B$. sanguinea, obtienen los mayores valores de dominancia relativa $(22.50$ y 11.73 , respectivamente). En cuanto a la frecuencia relativa $A$. acuminata, V. stipularis, Prunus serotina y Xylosma spiculifera alcanzan los valores más altos $(6.00$, respectivamente) (Tab. 7).

\section{Valor de Importancia de familia (VIF)}

En el bosque regenerado hay representadas un total de 34 familias, las familias más abundantes en todo el bosque fueron Solanaceae y Betulaceae, estas en conjunto representaron cerca del 70\% del número total de individuos. Fagaceae, Asteraceae, Elaeocarpaceae, Verbenaceae, Myrsinaceae y Fabaceae fueron otras familias representativas teniendo en cuenta el número de individuos restante (Tabla 8).

Solanaceae presentó un total de 1746 individuos, los cuales, estuvieron presentes en todas las parcelas excepto en P5; entre estos el mayor valor de importancia de familia fue para P4 (144.24) con un total de 306 individuos y P6 (78.99) con 601 individuos respectivamente. En el bosque regenerado la familia Solanaceae está representada por: S. ovalifolium, B. sanguinea, C. elegans y Salpichroa tristis, entre otras especies que no se tuvieron en cuenta para el cálculo como Solanum tuberosum y Physalis peruviana; es decir, esta familia se encuentra representada con el mayor número de especies dentro del bosque lo que la hace obtener los mayores valores para los parámetros fisionómicos necesarios para la obtención del VIF.

La familia Betulaceae, con un total de 90 individuos sembrados hace 16 años, se encontró en todas 
Tabla 1. Índice de Predominio Fisionómico, Parcela 1

\begin{tabular}{|c|c|c|c|c|}
\hline Especie & $\begin{array}{l}\text { Dominancia } \\
\text { relativa }\end{array}$ & $\begin{array}{c}\text { Cobertura } \\
\text { relativa }\end{array}$ & $\begin{array}{c}\text { Densidad } \\
\text { relativa }\end{array}$ & IPF \\
\hline Quercus humboldtii & 34.98 & 49.83 & 23.24 & 108.05 \\
\hline Alnus acuminata & 34.26 & 11.52 & 9.19 & 58.97 \\
\hline Solanum ovalifolium & 0.24 & 13.62 & 31.35 & 45.22 \\
\hline Fraxinus chinensis & 6.03 & 9.83 & 4.32 & 20.19 \\
\hline Baccharis latifolia & 11.36 & 4.13 & 1.62 & 17.11 \\
\hline Vallea stipularis & 8.06 & 2.79 & 4.32 & 15.17 \\
\hline Myrsine coriacea & 0.052 & 1.48 & 6,49 & 8.02 \\
\hline Prunus serotina & 0.034 & 1.78 & 4.32 & 6.15 \\
\hline Carica pubescens & 0.78 & 1.54 & 1.62 & 3.95 \\
\hline Myrcianthes leucoxyla & 0.026 & 0.48 & 3.24 & 3.75 \\
\hline Lantana sp. & 0.021 & 0.52 & 2.70 & 3.24 \\
\hline Cestrum elegans & 0.021 & 0.41 & 2.70 & 3.14 \\
\hline Xylosma spiculifera & 0.044 & 0.69 & 0.54 & 1.27 \\
\hline Oreopanax floribundum & 0.004 & 0.34 & 0.54 & 0.88 \\
\hline Piper bogotense & 0.004 & 0.34 & 0.54 & 0.88 \\
\hline Weinmannia tomentosa & 0.044 & 0.17 & 0.54 & 0.75 \\
\hline Inga sp. & 0.004 & 0.17 & 0.54 & 0.71 \\
\hline Myrsine guianensis & 0.004 & 0.17 & 0.54 & 0.71 \\
\hline Miconia squamulosa & 0.004 & 0.10 & 0.54 & 0.64 \\
\hline Clusia multiflora & 0.004 & 0.03 & 0.54 & 0.57 \\
\hline \multirow[t]{2}{*}{ Cordia cylindrostachya } & 0.004 & 0.03 & 0.54 & 0.57 \\
\hline & 100 & 100 & 100 & 300 \\
\hline
\end{tabular}


Tabla 2. Índice de Predominio Fisionómico, Parcela 2

\begin{tabular}{|c|c|c|c|c|}
\hline Especie & $\begin{array}{l}\text { Dominancia } \\
\text { relativa }\end{array}$ & $\begin{array}{l}\text { Cobertura } \\
\text { relativa }\end{array}$ & $\begin{array}{l}\text { Densidad } \\
\text { relativa }\end{array}$ & IPF \\
\hline Alnus acuminata & 27.74 & 45.65 & 15.27 & 88.66 \\
\hline Baccharis latifolia & 41.60 & 7.93 & 5.34 & 54.88 \\
\hline Vallea stipularis & 21.86 & 12.39 & 6.87 & 41.12 \\
\hline Solanum ovalifolium & 0.84 & 11.11 & 26.72 & 38.66 \\
\hline Myrsine guianensis & 1.18 & 3.18 & 9.16 & 13.52 \\
\hline Quercus humboldtii & 3.03 & 4.58 & 5.34 & 12.96 \\
\hline Lantana sp. & 0.13 & 6.31 & 5.34 & 12.96 \\
\hline Salpichroa tristis & 0.32 & 5.23 & 5.34 & 10.90 \\
\hline Myrcianthes leucoxyla & 0.09 & 0.51 & 7.63 & 8.23 \\
\hline Prunus serotina & 0.16 & 1.88 & 3.05 & 5.09 \\
\hline Montanoa quadrangularis & 0.02 & 0.41 & 4.58 & 5.02 \\
\hline Cestrum elegans & 0.01 & 0.18 & 2.29 & 2.48 \\
\hline Carica pubescens & 0.19 & 0.39 & 1.53 & 2.11 \\
\hline Xylosma spiculifera & 0.09 & 0.19 & 0.76 & 1.05 \\
\hline \multirow[t]{2}{*}{ Duranta mutisii } & 0.04 & 0.04 & 0.76 & 0.81 \\
\hline & 100 & 100 & 100 & 300 \\
\hline
\end{tabular}

las parcelas excepto en P4, su mayor VIF se presentó en P3 (94.33). Por su parte Fagaceae se encontró en P1 y P4 (61.99 y 16.60, con 72 y 2 individuos, respectivamente) y Asteraceae se encontró en P2 y P4 (62 y 48.48 con 14 y 37 respectivamente). Elaeocarpaceae, Verbenaceae, Myrsinaceae y Fabaceae se encontraron solamente en una parcela.

\section{DISCUSIÓN}

En el bosque se lograron identificar cinco estratos: rasante, herbáceo, arbustivo, subarbóreo y arbóreo inferior, en donde el estrato dominante fue el arbustivo. De acuerdo a los parámetros estructurales evaluados, los individuos que 
Tabla 3. Índice de Predominio Fisionómico, Parcela 3

\begin{tabular}{|c|c|c|c|c|}
\hline Especie & $\begin{array}{l}\text { Dominancia } \\
\text { relativa }\end{array}$ & $\begin{array}{c}\text { Cobertura } \\
\text { relativa }\end{array}$ & $\begin{array}{c}\text { Densidad } \\
\text { relativa }\end{array}$ & IPF \\
\hline Alnus acuminata & 63.89 & 33.81 & 25 & 123 \\
\hline Vallea stipularis & 9.47 & 20.97 & 27.71 & 58.16 \\
\hline Fraxinus chinensis & 1.99 & 10.06 & 7.23 & 19.28 \\
\hline Myrsine guianensis & 8.58 & 4.46 & 4.82 & 17.87 \\
\hline Solanum ovalifolium & 2.07 & 6.02 & 7.23 & 15.32 \\
\hline Citharexylum subflavescens & 4.49 & 5.8 & 1.28 & 11.49 \\
\hline Baccharis latifolia & 5.44 & 3.65 & 1.20 & 10.29 \\
\hline Cestrum elegans & 0.02 & 1.88 & 6.02 & 7.92 \\
\hline Clusia multiflora & 0.70 & 2.39 & 4.82 & 7.91 \\
\hline Bomarea multiflora & 0.51 & 3.92 & 2.41 & 6.85 \\
\hline Weinmannia tomentosa & 0.91 & 3.40 & 2.41 & 6.71 \\
\hline Myrcianthes leucoxyla & 1.17 & 1.74 & 1.20 & 4.11 \\
\hline Xylosma spiculifera & 0.06 & 0.29 & 2.41 & 2.76 \\
\hline Piper bogotense & 0.11 & 1.17 & 1.20 & 2.48 \\
\hline Cedrela montana & 0.01 & 0.08 & 2.41 & 2.50 \\
\hline Anacardiaceae indet. & 0.01 & 0.2 & 1.20 & 1.41 \\
\hline \multirow[t]{2}{*}{ Prunus serotina } & 0.01 & 0.13 & 1.20 & 1.34 \\
\hline & 100 & 100 & 100 & 300 \\
\hline
\end{tabular}




\begin{tabular}{|c|c|c|c|c|}
\hline Especie & $\begin{array}{l}\text { Dominancia } \\
\text { relativa }\end{array}$ & $\begin{array}{l}\text { Cobertura } \\
\text { relativa }\end{array}$ & $\begin{array}{l}\text { Densidad } \\
\text { relativa }\end{array}$ & IPF \\
\hline Brugmansia sanguinea & 65.19 & 55.54 & 48.85 & 169.50 \\
\hline Montanoa quadrangularis & 11.32 & 14.80 & 16.67 & 42.79 \\
\hline Cestrum elegans & 0.07 & 6.73 & 13.22 & 20.02 \\
\hline Quercus humboldtii & 9.61 & 4.86 & 1.15 & 15.62 \\
\hline Alnus acuminata & 4.27 & 6.06 & 3.43 & 13.75 \\
\hline Baccharis latifolia & 7.23 & 1.67 & 1.72 & 10.64 \\
\hline Retrophyllum rospigliosii & 0.29 & 3.79 & 6.29 & 10.37 \\
\hline Escallonia paniculata & 0.01 & 0.03 & 0.57 & 0.60 \\
\hline Myrcianthes leucoxyla & 0.54 & 1.51 & 2.30 & 4.35 \\
\hline Prunus serotina & 0.08 & 1.20 & 2.87 & 4.17 \\
\hline Fraxinus chinensis & 1.26 & 0.67 & 1.15 & 3.09 \\
\hline Vallea stipularis & 0.11 & 0.83 & 0.57 & 1.52 \\
\hline Xylosma spiculifera & 0.09 & 0.12 & 0.57 & 0.70 \\
\hline \multirow[t]{2}{*}{ Oreopanax floribundum } & 0.01 & 0.42 & 0.50 & 0.99 \\
\hline & 100 & 100 & 100 & 300 \\
\hline
\end{tabular}


Tabla 5. Índice de Predominio Fisionómico, Parcela 5

\begin{tabular}{ccccc}
\hline Especie & $\begin{array}{c}\text { Dominancia } \\
\text { relativa }\end{array}$ & $\begin{array}{c}\text { Cobertura } \\
\text { relativa }\end{array}$ & $\begin{array}{c}\text { Densidad } \\
\text { relativa }\end{array}$ & IPF \\
\hline Citharexylum subflavescens & 29.62 & 30.68 & 18.18 & 78.49 \\
Alnus acuminata & 24.93 & 28.40 & 25 & 78.33 \\
Myrsine coriacea & 9.01 & 11.80 & 20.45 & 41.27 \\
Xylosma spiculifera & 2.29 & 8.26 & 13.64 & 24.19 \\
\hline Oreopanax floribundum & 12.71 & 5.11 & 4.55 & 22.37 \\
Cedrela montana & 16.42 & 3.53 & 2.27 & 13.24 \\
Duranta mutisii & 4.69 & 6.28 & 2.27 & 3.20 \\
Vallea stipularis & 0.02 & 2.36 & 6.82 & 3.85 \\
\hline Prunus serotina & 0.27 & 1.57 & 2.27 & 3.75 \\
Sambucus nigra & 0.01 & 1.57 & 2.17 & 2.69 \\
\hline Morella pubescens & 0.03 & 0.39 & 100 & 300 \\
\hline
\end{tabular}

predominaron en el ecosistema, se encontraron con mayor frecuencia en los primeros intervalos de clase, lo que coincide con los resultados que se presentaron en bosques maduros circundantes, como el Cerro de Majuy (Cortés, 2003).

Dieciséis años después de haber sido sembrado el bosque, la estructura de la vegetación presenta, por distribución de frecuencias, de los intervalos de clase de altura, diámetro y cobertura, una forma de $J$ invertida, que es una tendencia en los ecosistemas de referencia evaluados y es, además, característico de áreas que se encuentran en buen estado de desarroIlo (Hernández-Stefanoni et al, 2011), ya que demuestra que se está presentando regeneración natural, estratificación y conformación de un estrato de juveniles que están integrando el sotobosque.

La tendencia de la curva de la J invertida también señala que las comunidades vegetales que se 


\begin{tabular}{|c|c|c|c|c|}
\hline Especie & $\begin{array}{c}\text { Dominancia } \\
\text { relativa }\end{array}$ & $\begin{array}{l}\text { Cobertura } \\
\text { relativa }\end{array}$ & $\begin{array}{l}\text { Densidad } \\
\text { relativa }\end{array}$ & IPF \\
\hline Solanum ovalifolium & 20.88 & 39.34 & 45.53 & 105.75 \\
\hline Alnus acuminata & 35.53 & 23.31 & 17.07 & 75.91 \\
\hline Baccharis latifolia & 9.54 & 6.66 & 3.25 & 19.45 \\
\hline Vallea stipularis & 8.89 & 4.61 & 3.25 & 16.75 \\
\hline Senna viarum & 7.39 & 4.87 & 2.44 & 14.70 \\
\hline Myrcianthes leucoxyla & 2.73 & 3.13 & 5.69 & 11.55 \\
\hline Ulex europaeus & 6.26 & 2.46 & 1.63 & 10.35 \\
\hline Weinmannia tomentosa & 3.29 & 4.61 & 2.44 & 10.33 \\
\hline Prunus serotina & 0.62 & 2.36 & 5.69 & 8.66 \\
\hline Myrsine guianensis & 2.48 & 2.36 & 1.63 & 6.46 \\
\hline Xylosma spiculifera & 0.60 & 2.82 & 3.25 & 6.67 \\
\hline Rubus glaucus & 0.02 & 0.46 & 2.44 & 2.92 \\
\hline Fraxinus chinensis & 1.05 & 1.02 & 0.81 & 2.88 \\
\hline Miconia squamulosa & 0.01 & 0.46 & 1.63 & 2.10 \\
\hline Cestrum elegans & 0.01 & 0.75 & 0.81 & 1.57 \\
\hline Carica pubescens & 0.50 & 0.25 & 0.81 & 1.56 \\
\hline Citharexylum subflavescens & 0.10 & 0.25 & 0.81 & 1.16 \\
\hline \multirow[t]{2}{*}{ Retrophyllum rospigliosii } & 0.10 & 0.25 & 0.81 & 1.16 \\
\hline & 100 & 100 & 100 & 300 \\
\hline
\end{tabular}


Tabla 7. Índice de Valor de Importancia

\begin{tabular}{|c|c|c|c|c|}
\hline Especie & $\begin{array}{c}\text { Densidad } \\
\text { relativa }\end{array}$ & $\begin{array}{c}\text { Dominancia } \\
\text { relativa }\end{array}$ & $\begin{array}{c}\text { Frecuencia } \\
\text { relativa }\end{array}$ & IVI \\
\hline Solanum ovalifolium & 55,22 & 2,38 & 4,00 & 61,60 \\
\hline Alnus acuminata & 4,01 & 22,50 & 6,00 & 32,51 \\
\hline Brugmansia sanguinea & 5,26 & 11,73 & 1,00 & 17,99 \\
\hline Cestrum elegans & 11,74 & 0,90 & 5,00 & 17,64 \\
\hline Vallea stipularis & 3,63 & 7,02 & 6,00 & 16,65 \\
\hline Baccharis latifolia & 0,75 & 9,74 & 5,00 & 15,49 \\
\hline Fraxinus chinensis & 0,71 & 9,32 & 4,00 & 14,03 \\
\hline Quercus humboldtii & 3,38 & 6,88 & 3,00 & 13,27 \\
\hline Myrcianthes leucoxyla & 1,59 & 4,69 & 5,00 & 11,27 \\
\hline Ulex europaeus & 0,17 & 9,74 & 1,00 & 10,91 \\
\hline Myrsine coriacea & 2,05 & 5,04 & 2,00 & 9,08 \\
\hline Myrsine guianensis & 2,63 & 1,63 & 4,00 & 8,26 \\
\hline Prunus serotina & 1,21 & 0,14 & 6,00 & 7,35 \\
\hline Xylosma spiculifera & 0,63 & 0,46 & 6,00 & 7,09 \\
\hline Citharexylum subflavescens & 0,42 & 3,04 & 3,00 & 6,46 \\
\hline Lantana sp. & 0,88 & 1,02 & 3,00 & 4,90 \\
\hline Weinmannia tomentosa & 0,25 & 0,96 & 3,00 & 4,21 \\
\hline Oreopanax floribundum & 0,79 & 0,02 & 3,00 & 3,81 \\
\hline Montanoa quadrangularis & 1,71 & 0,02 & 2,00 & 3,73 \\
\hline Duranta mutisii & 0,17 & 0,36 & 3,00 & 3,52 \\
\hline Carica pubescens & 0,25 & 0,18 & 3,00 & 3,43 \\
\hline Cedrela montana & 0,17 & 1,25 & 2,00 & 3,42 \\
\hline
\end{tabular}




\begin{tabular}{|c|c|c|c|c|}
\hline & & \multirow[b]{2}{*}{0,00} & \multirow{2}{*}{3,00} & \multirow[b]{2}{*}{3,29} \\
\hline Monnina salicifolia & 0,29 & & & \\
\hline Retrophylum rospigliosii & 0,50 & 0,07 & 2,00 & 2,57 \\
\hline Miconia squamulosa & 0,42 & 0,00 & 2,00 & 2,42 \\
\hline Clusia multiflora & 0,21 & 0,12 & 2,00 & 2,32 \\
\hline Piper bogotense & 0,08 & 0,02 & 2,00 & 2,10 \\
\hline Senna viarum & 0,13 & 0,67 & 1,00 & 1,79 \\
\hline Salpichroa tristis & 0,33 & 0,05 & 1,00 & 1,38 \\
\hline Inga sp. & 0,08 & 0,00 & 1,00 & 1,08 \\
\hline Morella pubescens & 0,04 & 0,00 & 1,00 & 1,04 \\
\hline Anacardiaceae (Indet) & 0,04 & 0,00 & 1,00 & 1,04 \\
\hline Cordia cylindrostachya & 0,04 & 0,00 & 1,00 & 1,04 \\
\hline Escallonia paniculata & 0,04 & 0,00 & 1,00 & 1,04 \\
\hline \multirow[t]{2}{*}{ Sambucus nigra } & 0,04 & 0,00 & 1,00 & 1,04 \\
\hline & 100 & 100 & 100 & 300 \\
\hline
\end{tabular}

conformaron en el bosque se encuentra en coherente proceso de desarrollo en dirección a etapas sucesionales de crecimiento y productividad vegetal más avanzadas, con relación a la abundancia de individuos jóvenes que, por supuesto, bajo las condiciones apropiadas, reemplazarán a individuos arbóreos pertenecientes a las clases diamétricas superiores que ya se encuentran en fases de deterioro avanzado o mortalidad (Arruda et al, 2011).

Las líneas sucesionales de vegetación del bosque regenerado, han sido definidas por las especies climácicas seleccionadas en la siembra inicial, como fueron $A$. acuminata y $Q$. humboldtii, las cuales, a mediano plazo, promovieron el restablecimiento de la cobertura característica de un bosque maduro, lo que en la actualidad constituye el $54 \%$ de la cobertura de los estratos superiores del bosque regenerado.

Las condiciones que se presentan en el bosque regenerado han favorecido el éxito ecológico de $S$. ovalifolium, dentro de la sucesión, ya que, a pesar de no haber sido sembrada, ha establecido individuos juveniles aprovechando las condiciones para crecer; así mismo, las familias Solanaceae y Asteraceae se han visto beneficiadas, creando las condiciones de sobrevivencia que las han favorecido frente al resto de la comunidad vegetal. 
Tabla 8. Valor de Importancia de familia.

\begin{tabular}{|c|c|c|c|}
\hline Parcela & $\begin{array}{l}\text { Familia } \\
\text { botánica }\end{array}$ & VIF & $\begin{array}{l}\text { Número de } \\
\text { individuos }\end{array}$ \\
\hline \multirow{3}{*}{1} & Fagaceae & 61.99 & 72 \\
\hline & Betulaceae & 51.46 & 17 \\
\hline & Solanaceae & 42.15 & 588 \\
\hline \multirow{3}{*}{2} & Betulaceae & 47.5 & 20 \\
\hline & Asteraceae & 62 & 14 \\
\hline & Solanaceae & 66.84 & 166 \\
\hline \multirow{3}{*}{3} & Betulaceae & 94.33 & 21 \\
\hline & Elaeocarpaceae & 42.49 & 56 \\
\hline & Solanaceae & 46.3 & 85 \\
\hline \multirow{3}{*}{4} & Solanaceae & 144.24 & 306 \\
\hline & Asteraceae & 48.48 & 37 \\
\hline & Fagaceae & 16.6 & 2 \\
\hline \multirow{3}{*}{5} & Betulaceae & 55.96 & 11 \\
\hline & Verbenaceae & 68.14 & 9 \\
\hline & Myrsinaceae & 35.71 & 1 \\
\hline \multirow{3}{*}{6} & Solanaceae & 78.99 & 601 \\
\hline & Betulaceae & 58.49 & 21 \\
\hline & Fabaceae & 29.49 & 7 \\
\hline
\end{tabular}


Los individuos juveniles presentes en el bosque regenerado evidencian que el bosque es capaz de garantizar la reproducción de las poblaciones de especies nativas que fueron sembradas y de especies que llegaron espontáneamente, que aseguran su desarrollo y continuidad. Este estudio permitió demostrar que el bosque regenerado ha sido lo suficientemente resiliente, 16 años luego de su establecimiento, ya que ha soportado satisfactoriamente periodos de estrés de origen natural y antropogénico, independiente de las variaciones climáticas reportadas para la cuenca alta del Río Bogotá (Cortés et al, 1999), lo que le permite mantener las características que se evidenciaron al momento del estudio.

Así mismo, existen estudios que demuestran la presencia de especies de la fauna local, representada por aves (Echeverry y Morales, 2007) reptiles y mamíferos (observaciones directas en campo), que probablemente se comparten e intercambian con el del ecosistema de referencia más cercano, el caso del Cerro de Majuy (Cortés et al, 1999; Cortés, 2003).

\section{CONCLUSIONES}

En general, el plan de rehabilitación a partir de la siembra de especies nativas implementado en éste estudio fue adecuado ya que se pudo observar vigor en la totalidad de los individuos que fueron sembrados, los cuales dominaron los estratos arbóreo y arbustivo. El bosque regenerado, presentó individuos en los cinco estratos de vegetación, lo que sugiere una similitud estructural, comparados con los resultados de Cortés (2003) para los bosques de base de montaña del Cerro de Majuy.

Las especies juveniles, que surgieron a partir de la siembra inicial, garantizan la propagación, crecimiento y el desarrollo del bosque regenerado. Además la cercanía a una fuente importante de propágulos como lo son los bosques y matorrales del cerro de
Majuy, hacen que se garantice y se proyecte de manera natural la riqueza del bosque, asegurado además, por un flujo de dispersores y polinizadores provenientes de estas áreas más conservadas, pese a los elementos antrópicos que fragmentan el ecosistema. La aparición de especies de plantas nativas, producto de los mecanismos de dispersión zoócora, junto con la polinización de insectos y la presencia de especies espontáneas al interior del bosque regenerado, evidencian que existen mecanismos que facilitan el funcionamiento de sus estados ecológicos de desarrollo.

El bosque regenerado en 16 años alcanzó a presentar parte de los elementos dominantes de los ecosistemas de referencia con los que fue planeado y comparado, independiente del impacto del cambio climático y de la creciente expansión urbanística en Chía, por lo que se asume que este logrará alcanzar parte del arreglo característico de bosques de base de montaña y de planicie de la Sabana de Bogotá.

El proyecto diseñado por el Profesor Thomas van der Hammen comprueba el potencial de trabajo recíproco social y ecosistémico pues, al crear las condiciones favorables para la rehabilitación natural, se convierte un área degradada en un parche de vegetación autosostenible, en el cual el mismo bosque se encarga de completar su regeneración natural y, posterior, restauración ecológica.

\section{AGRADECIMIENTOS}

Los autores agradecen a la familia van der Hammen quienes nos proporcionaron la información y asesoraron el desarrollo de este trabajo. Además, a la Universidad Nacional de Colombia y Universidad Militar "Nueva Granada" por su gentil auspicio. Muy especialmente agradecemos al Profesor Thomas van der Hammen (q.e.p.d.) quien asesoró, hizo seguimiento al trabajo de campo y revisó los resultados finales de esta investigación. 


\section{REFERENCIAS}

1 Arruda, D. M., D. O. Brandao, F. V. Costa, G. S. Tolentino, R. D. Brasil, S. N. D'ângeloneto., Y. R. Nunes-Ferreira. 2011. Structural aspects and floristic similarity among tropical dry forest fragments with different management histories in Northern Minas Gerais, Brazil. Revista Árvore 35: 131-142.

2 Brasher, A. 2003. Impacts of human disturbances on biotic communities in Hawaiian streams. BioScience 53(11): 1052-1060.

3 Castellanos, L. I. 2011. Conocimiento etnobotánico, patrones de uso y manejo de plantas útiles en la cuenca del Río Cane - Iguaque (Boyacá - Colombia); una aproximación desde los sistemas de uso de la biodiversidad. Ambiente \& Sociedade 14(1): 45-75.

4 Claro, F. A. 1995. Estudio Agroclimático de la Cuenca Alta del Río Bogotá y del Río UbatéSuárez. IDEAM. Bogotá D.C. 120 p.

5 Cortés, S. P. 1997. Contribuciones al estudio de la diversidad florística en el sector cerro Manjuy, parte baja de la Cuenca del Río Frío, Municipio de Chía. Trabajo de grado. Departamento de Biología, Universidad Nacional de Colombia (inédito). Bogotá D.C. 148pp.

6 Cortés, S. P. 2003. Estructura de la vegetación arbórea y arbustiva en el costado oriental de la Serranía de Chía (Cundinamarca, Colombia). Caldasia 25 (1): 119-137.
7 Cortés, S. P., T, van der Hammen., J. O., Rangel. 1999. Comunidades vegetales y patrones de degradación y sucesión en la vegetación de los Cerros Occidentales de Chía-Cundinamarca-Colombia. Revista de la Academia Colombiana de Ciencias Exactas, Físicas y Naturales 23 (89): 529-554.

8 Echeverry, M. A., A. Morales. 2007. Listado anotada de alguna especies de la vereda "Cerca de Piedra", Chía, Colombia. Departamento de Biología, Pontificia Universidad Javeriana, Bogotá, Colombia. Boletín de la Sociedad Antioqueña de Ornitología (SAO) XVII. Bogotá D.C. 6 p.

9 Finol, H. 1976. Estudio fitosociológico de las unidades 2 y 3 de la Reserva Forestal de Carapo, Estado de Barinas. Acta Botánica Venezuelica 10(1-4): 15-103.

10 Hernández-Stefanoni, J. L., J. M. Dupuy, F. TunDzul \& F. May-Pat. 2011. Influence of landscape structure and stand age on species density and biomass of a tropical dry forest across spatial scales. Landscape Ecology 26: 355-370.

11 Judd, W. S., C, Campbell., E, Kellog. 2002. Plant Systematics: a phylogenetic approach. Editorial Sinauer. Massachusetts -USA. 464 p.

12 Londoño, V., A, Torres. 2015. Estructura y composición vegetal de un bosque seco tropical en regeneración en Bataclán (Cali, Colombia). Revista Colombia Forestal 18(1): 71-85. 
13 Lozano, F; E, Jiménez; Caicedo, P. 2006. Simposio - Conservación de biodiversidad en paisajes rurales: aprendizajes en la cuenca media del Río Barbas (Filandia, Quindío). En: Andrade, M. G; J, Aguirre; J. V. Rodríguez-Mahecha (eds.). Libro de resúmenes II Congreso Colombiano de Zoología. p 43-48. Editorial Panamericana Formas e Impresos S. A. 572 p.

14 MAVDT. 1999. Programa para la implementación del plan estratégico para la restauración de bosque en Colombia - Plan Verde. Ministerio de Ambiente, Vivienda y Desarrollo Territorial. Bogotá D.C. 17 p.

15 Mori, S., B, Boom. 1983. Ecological importance of Myrtaceae in an Eastern Brazilian forest. Biotropica 15 (1): 68-70.

16 POT. 2000. Plan de Ordenamiento Territorial del Municipio de Chía (Cundinamarca). Alcaldía Municipal de Chía. Chía-Cundinamarca. $135 \mathrm{p}$.

17 Rangel, J. O., A, Garzón. 1994. Aspectos de la estructura, de la diversidad y de la dinámica de la vegetación del Parque Regional de Ucumarí. En J.O. Rangel (ed). Ucumari un caso típico de La Diversidad Biótica Andina. p 85-108. Publicaciones de la CARDER. Pereira.

18 Rangel, J. O., G, Lozano. 1986. Un perfil de vegetación entre La Plata (Huila) y el Volcán Puracé. Caldasia 14(68-70): 53-547.
19 Rangel, J. O., A, Velázquez. 1997. Métodos de estudio de la vegetación. En: Rangel, J. O. Colombia Diversidad Biótica II. Instituto de Ciencias Naturales, Universidad Nacional de Colombia, IDEAM. p 59-87. Bogotá D.C.

20 Samper, C. 1999. Ecosistemas naturales, restauración ecológica e investigación. En: Ponce de León, E. Restauración ecológica y reforestación. Fundación Alejandro Ángel Escobar, Fundación Friedrich Ebert de Colombia - Fescol, Foro Nacional Ambiental, GTZ. p 53-81. Bogotá D.C.

21 Saunders, D. A., R. J., Hobbs., C. R. Margules. 1991. Biological Consequences of Ecosystem Fragmentation: A Review. Conservation Biology 5(1): 18-32.

22 Van der Hammen, T. 1998. Plan Ambiental de la Cuenca Alta del Río Bogotá. Corporación Autónoma Regional de Cundinamarca (CAR). Bogotá D.C. 142 p.

23 van der Hammen, T. 1999. Consensos mundiales de restauración y enfoques de investigación y monitoreo. En: Ponce de León, E. Restauración ecológica y reforestación. Fundación Alejandro Ángel Escobar, Fundación Friedrich Ebert de Colombia - Fescol, Foro Nacional Ambiental, GTZ. p 53-81. Bogotá D.C.

24 van der Hammen, T., B, van Geel. 1982. Historia geológica y secuencia vegetacional y climática de la Cordillera Oriental. Universidad Nacional de Colombia, Revista Geografía 3: 225-231. 